\section{(6) OPEN ACCESS}

\title{
Paracetamol: not as safe as we thought? A systematic literature review of observational studies
}

\author{
Emmert Roberts, ${ }^{1}$ Vanessa Delgado Nunes, ${ }^{2}$ Sara Buckner, ${ }^{2}$ Susan Latchem, ${ }^{2}$ \\ Margaret Constanti, ${ }^{2}$ Paul Miller, ${ }^{2}$ Michael Doherty, ${ }^{3}$ Weiya Zhang, $^{3}$ Fraser Birrell, ${ }^{4}$ \\ Mark Porcheret, ${ }^{5}$ Krysia Dziedzic, ${ }^{6}$ Ian Bernstein, ${ }^{7,8}$ Elspeth Wise, ${ }^{9}$ \\ Philip G Conaghan ${ }^{10}$
}

\begin{abstract}
Handling editor Tore K Kvien
- Additional material is

published online only. To view please visit the journal online (http://dx.doi.org/10.1136/ annrheumdis-2014-206914).
\end{abstract}

For numbered affiliations see end of article.

\section{Correspondence to} Professor Philip G Conaghan Leeds Institute of Rheumatic and Musculoskeletal Medicine, Chapel Allerton Hospital, Leeds LS7 4SA, UK; p.conaghan@leeds.ac.uk

Received 31 October 2014 Revised 4 December 2014 Accepted 13 January 2015 Published Online First 2 March 2015

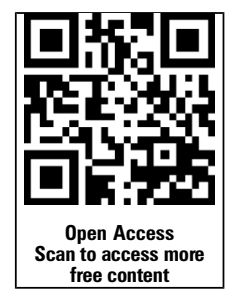

CrossMark

\begin{tabular}{|l|}
\hline To cite: Roberts E, Delgado \\
Nunes V, Buckner S, et al. \\
Ann Rheum Dis \\
2016;75:552-559. \\
\hline
\end{tabular}

\section{ABSTRACT}

Objectives We conducted a systematic literature review to assess the adverse event (AE) profile of paracetamol. Methods We searched Medline and Embase from database inception to 1 May 2013. We screened for observational studies in English, which reported mortality, cardiovascular, gastrointestinal (GI) or renal AEs in the general adult population at standard analgesic doses of paracetamol. Study quality was assessed using Grading of Recommendations Assessment, Development and Evaluation. Pooled or adjusted summary statistics were presented for each outcome.

Results Of 1888 studies retrieved, 8 met inclusion criteria, and all were cohort studies. Comparing paracetamol use versus no use, of two studies reporting mortality one showed a dose-response and reported an increased relative rate of mortality from 0.95 (0.92 to 0.98 ) to 1.63 (1.58 to 1.68). Of four studies reporting cardiovascular AEs, all showed a dose-response with one reporting an increased risk ratio of all cardiovascular AEs from 1.19 (0.81 to 1.75$)$ to 1.68 (1.10 to 2.57). One study reporting GI AEs reported a dose-response with increased relative rate of $\mathrm{GI}$ AEs or bleeds from 1.11 (1.04 to 1.18 ) to 1.49 (1.34 to 1.66). Of four studies reporting renal AEs, three reported a doseresponse with one reporting an increasing OR of $\geq 30 \%$ decrease in estimated glomerular filtration rate from 1.40 (0.79 to 2.48 ) to 2.19 (1.4 to 3.43 ).

Discussion Given the observational nature of the data, channelling bias may have had an important impact. However, the dose-response seen for most endpoints suggests a considerable degree of paracetamol toxicity especially at the upper end of standard analgesic doses.

\section{INTRODUCTION}

Paracetamol is the most widely used over-thecounter and prescription analgesic worldwide. ${ }^{1}$ It is the first step on the WHO pain ladder and is currently recommended as first-line pharmacological therapy by a variety of international guidelines for a multitude of acute and chronic painful conditions. $^{2}$

The mechanism of paracetamol's analgesic action remains largely unknown, but recent studies demonstrate that paracetamol inhibits prostaglandin production within the central nervous system and within peripheral tissues. ${ }^{3}$ Irrespective of its efficacy, it is generally considered to be safer than other commonly used analgesics such as non-steroidal anti-inflammatory drugs (NSAIDs) or opiates.

The analgesic benefit of paracetamol has recently been called into question in the management of one chronic painful condition, osteoarthritis (OA). ${ }^{4}$ Clinicians and patients need up-to-date evidence of benefits and harms to make evidence-based decisions on pharmacological prescription, and a recent estimate of the true risks of paracetamol at standard analgesic doses has not been available. We therefore conducted a systematic review of studies investigating the association between paracetamol and major adverse events (AEs) in the general adult population to provide a clinically informative toxicity profile.

\section{METHODS}

\section{Data sources and study selection}

We followed recommendations made by the Metaanalysis of Observational Studies in Epidemiology and the Preferred Reporting Items for Systematic Reviews and Meta-Analyses (PRISMA) groups. ${ }^{5}$

We searched Medline and Embase for Englishlanguage studies published from database inception to 7 May 2013. The full search strategy was limited to only identify observational studies and can be found in the online supplementary material. All relevant references were checked for additional citations. Randomised controlled trial (RCT)-level evidence was not considered a meaningful way of capturing $\mathrm{AE}$ data because of the short-term follow-up of RCT trial participants as well as strict eligibility for trial entry, meaning that the general population would not be represented. If cohort-level evidence was found for an $\mathrm{AE}$ outcome, casecontrol evidence was not considered.

Studies were eligible for inclusion if they met the predefined protocol: the study population was adults aged $>18$ years and the study investigated one or more of the AEs of interest when people were taking oral paracetamol at a standard therapeutic dose of $0.5-1 \mathrm{~g}$ every $4-6 \mathrm{~h}$ to a maximum of $4 \mathrm{~g} /$ day compared with non-use.

\section{Outcomes}

The main outcomes investigated were all-cause mortality, cardiovascular AEs (specifically incidence of myocardial infarction, cerebrovascular accidents and hypertension), gastrointestinal (GI) AEs (specifically incidence of GI bleeding) and renal AEs 
(specifically reductions in estimated glomerular filtration rate (eGFR), increases in serum creatinine concentration and the need for renal replacement therapy).

We first screened titles and abstracts, and one reviewer (SB) screened relevant full-text articles. The second reviewer (VDN) reviewed $10 \%$ of the full-text articles screened, which were selected at random. One reviewer (SB) extracted study characteristics and adjusted summary statistics with $95 \% \mathrm{CIs}$ and recorded the data in a standard form. Two authors (SB and ER) independently assessed the study quality using Grading of Recommendations Assessment, Development and Evaluation (GRADE). Each outcome is given a quality rating of high, moderate, low or very low based upon risk of bias, inconsistency, indirectness and imprecision. Risk of bias for each outcome was assessed using checklists for observational studies, which are based on the Strengthening the Reporting of Observational Studies in Epidemiology statement. ${ }^{7}$ GRADE clinical evidence profiles for each outcome can be found in the online supplementary material.

\section{Statistical analysis}

Studies that met the inclusion criteria and reported summary statistics with $95 \%$ CIs, or presented sufficient data for the calculation of summary statistics and 95\% CIs, were considered for inclusion in meta-analysis. Where data were able to be pooled, heterogeneity was assessed using the $\chi^{2}$ and $\mathrm{I}^{2}$ statistics. Heterogeneity was predefined as $\chi^{2} \mathrm{p}<0.1$ or $\mathrm{I}^{2}>50 \%$, and where heterogeneity was unable to be removed by predefined subgroups a random effects model was assumed and outcomes were downgraded in quality. In instances where data were unable to be pooled, due to difference in outcome or paracetamol dosage reporting, individual adjusted summary statistics were presented for each outcome per study. We produced forest plots to visually assess the summary statistics and 95\% CIs of each study; analyses were done with Review Manager Version Five.

\section{RESULTS}

The search process identified 1888 records. Eight studies met the inclusion criteria, all of which were cohort studies. As all prespecified outcomes were found from these eight cohort studies, no case-control evidence was considered. Figure 1 shows the PRISMA flow chart for study selection. Table 1 reports the included study characteristics and results. The quality of evidence varied between outcomes and was graded as low or very low across all outcomes. Due to the non-comparable nature of outcomes and different paracetamol dosage definitions reported by individual studies, meta-analysis was only possible for a singular outcome; the incidence of hypertension. For all other outcomes, individual adjusted summary statistics are presented by AE category.

Two studies reported mortality, ${ }^{8} 9$ of which one reported a dose-response increase in the relative rate of all-cause mortality, ${ }^{8}$ and one reported a significantly increased standardised mortality ratio for those patients prescribed paracetamol compared with those not prescribed paracetamol as shown in figure $2 .{ }^{9}$

Four studies reported cardiovascular AEs, ${ }^{8}{ }^{10-12}$ all of which demonstrated a dose-response. One study reported a doseresponse increase in the risk ratio of cardiovascular AEs (confirmed or probable non-fatal myocardial infarction, non-fatal stroke, fatal coronary heart disease or fatal stroke) as shown in figure $3 ; 1^{10}$ one study reported a dose-response increase in the relative rate of the new cases of myocardial infarction and stroke ${ }^{8}$ and two studies reported a dose-response increase, which remained when data were pooled, in the relative risk of new cases of hypertension for those patients taking paracetamol compared with those not taking paracetamol. ${ }^{11} 12$

One study reported GI AEs, ${ }^{8}$ which showed a dose-response increase in the relative rate of upper GI AEs (gastroduodenal ulcers and complications such as upper GI haemorrhages) for those patients prescribed paracetamol compared with those not prescribed paracetamol as shown in figure 4 .

Four studies reported renal AEs, ${ }^{8}{ }^{13-15}$ three of which demonstrated a dose-response. One study reported a doseresponse increase in OR of a decrease of $\geq 30 \mathrm{~mL} / \mathrm{min} / 1.73 \mathrm{~m}^{2}$ in eGFR and $\geq 30 \%$ decrease in eGFR as shown in figure $5 ;^{13}$ one study reported a dose-response increase in the number of new cases of acute renal failure ${ }^{8}$ one study reported a doseresponse increase in OR of $\geq 0.3 \mathrm{mg} / \mathrm{dL}$ increase in serum creatinine and a decrease of $\geq 30 \mathrm{~mL} / \mathrm{min} / 1.73 \mathrm{~m}^{2}$ in eGFR; ${ }^{15}$ and one study reported no dose-response relationship in the estimated progression rates of chronic kidney disease and no difference in time to renal replacement therapy between those taking paracetamol and those not taking paracetamol. ${ }^{14}$

\section{DISCUSSION}

The objective of this review was to synthesise the long-term observational evidence of the harmful effects of paracetamol. Findings from this systematic review demonstrate a consistent dose-response relationship between paracetamol at standard analgesic doses and AEs that are often observed with NSAIDs. This includes a dose-response relationship between paracetamol and increasing incidence of mortality, cardiovascular, GI and renal AEs in the general adult population.

The main limitations of this study are the low number of studies and quality of available evidence. As all studies included were observational, the GRADE system of quality rating per outcome begins at 'low' quality and can subsequently be upgraded or downgraded per individual outcome. This initial 'low' rating is based purely on the study's observational designs and does not take into consideration that observational studies are the most appropriate study design to assess the risk of longterm $\mathrm{AE}$ outcomes. Five of the studies were conducted in healthy female registered nurses or male physicians, which may limit the generalisability of the data to the general population. ${ }^{10-13} 15$

Although the sample size of most included studies was large, and the reported dosage regimens were consistent with modern dosage regimens, reliance on self-reported medication use and channelling bias with incomplete adjustment for potential confounders may have had an important impact. Four studies did not adjust for concomitant NSAID use, and channelling bias may lead those patients deemed unsuitable for NSAID therapy to be prescribed paracetamol as a 'safer' alternative, ${ }^{8} 91214$ thus creating an allocation bias to a preselected group of patients with higher risk of AEs. Often referred to in the literature as 'confounding by indication', the indication and choice of analgesic treatment by clinicians may be related to the risk of future health outcomes and result in an imbalance of the underlying risk profile between paracetamol and non-paracetamol groups, potentially leading to biased results. All confounders adjusted for in each study can be found in the online supplementary material table S1. Six studies described selfreported medication use ${ }^{10-15}$ and two studies ${ }^{89}$ used only paracetamol prescription data. Both of these methods have the potential to inaccurately estimate the true amount of taken paracetamol in the studied cohort. A further limitation of the review is the various definitions of paracetamol dosage regimen across 


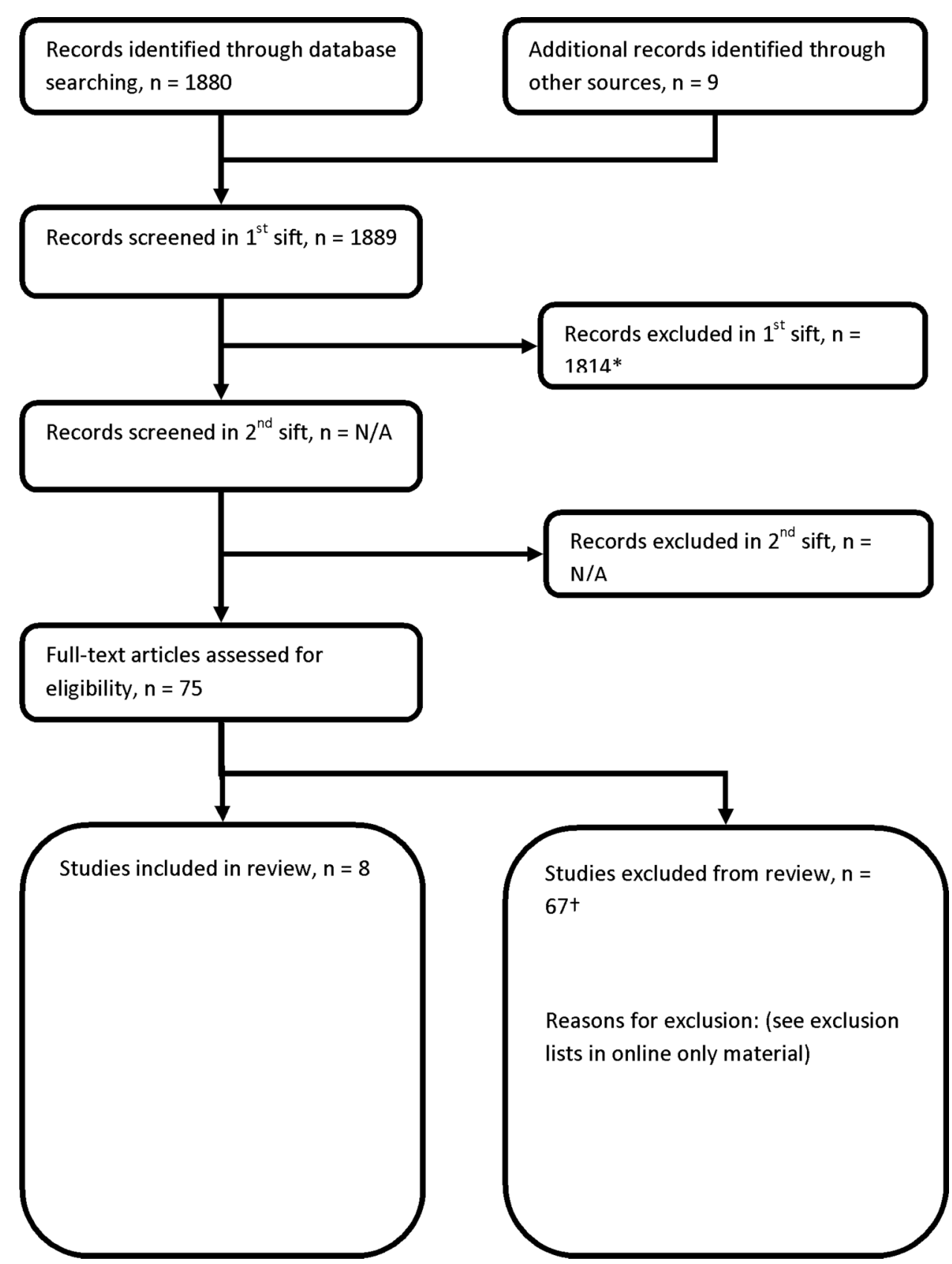

Figure 1 Study selection. *Included animal studies, non-biological science studies and human studies of paracetamol not reporting adverse events. tIncluded reviews, editorials and commentaries; types of study not in inclusion criteria; outcome measures other than those in inclusion criteria.

studies. Some report lifetime intake, while others report the number of pills or grams of paracetamol per unit time. This prevented the quantitative pooling of different doses in meta-analysis and the ability to draw firm conclusions as to safe dosage regimens.

While these limitations are important to consider, the striking trend of dose-response is consistent across multiple outcomes and studies. There is also evidence from the case-control literature supporting the dose-response seen in the current review, ${ }^{16}$ and a similar toxicity profile is demonstrated in systematic reviews of short-term RCTs. ${ }^{4}$

Several large observational studies confirm a better side effect profile for paracetamol compared with traditional NSAIDs. ${ }^{17} 18$ In line with the findings of the current review, one such study has also shown that the combination use of paracetamol and
NSAIDs significantly increased the number of hospitalisations for upper GI AEs. ${ }^{18}$ In keeping with our findings, the addition of gastroprotective agents to paracetamol prescription significantly reduced this event compared with paracetamol alone. As well, a recent RCT comparing paracetamol and ibuprofen in a population of patients with knee pain showed 14/192 (7\%) patients in the paracetamol arm experienced a haemoglobin drop of $\geq 1 \mathrm{~g} / \mathrm{dL}$ at day 10 , and by week 13 this figure rose to $20 \%,{ }^{19}$ which was not significantly different from the drops in haemoglobin observed in the ibuprofen-only group.

Every prescribing decision involves a calculation of risk versus benefit, a trade-off of efficacy versus tolerability. If providing adequate analgesia or antipyresis, clinicians and patients may be willing to accept the risk at the level of AEs demonstrated in this review. However, when analgesic benefit is uncertain, as has 
Table 1 Studies included in the review

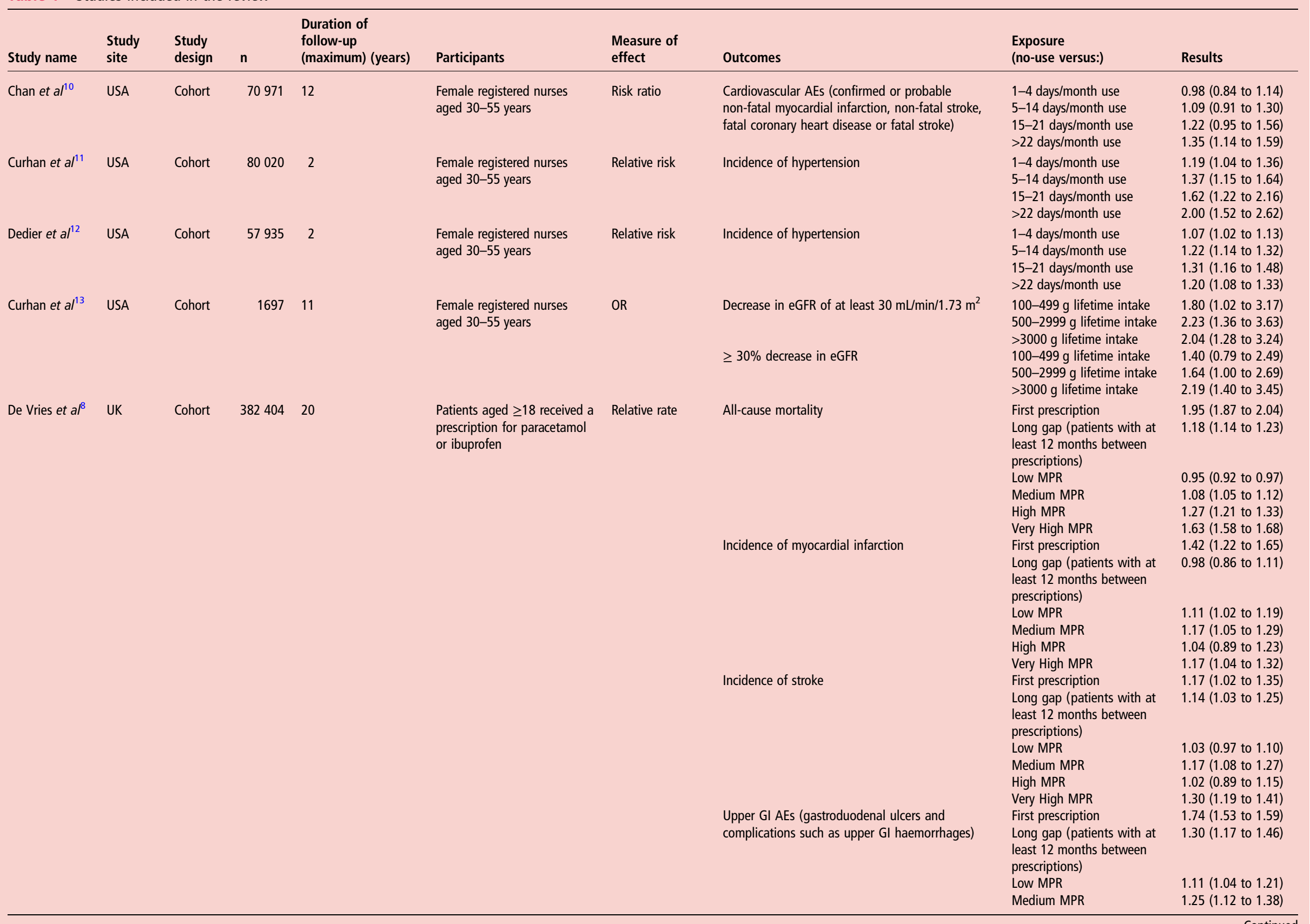




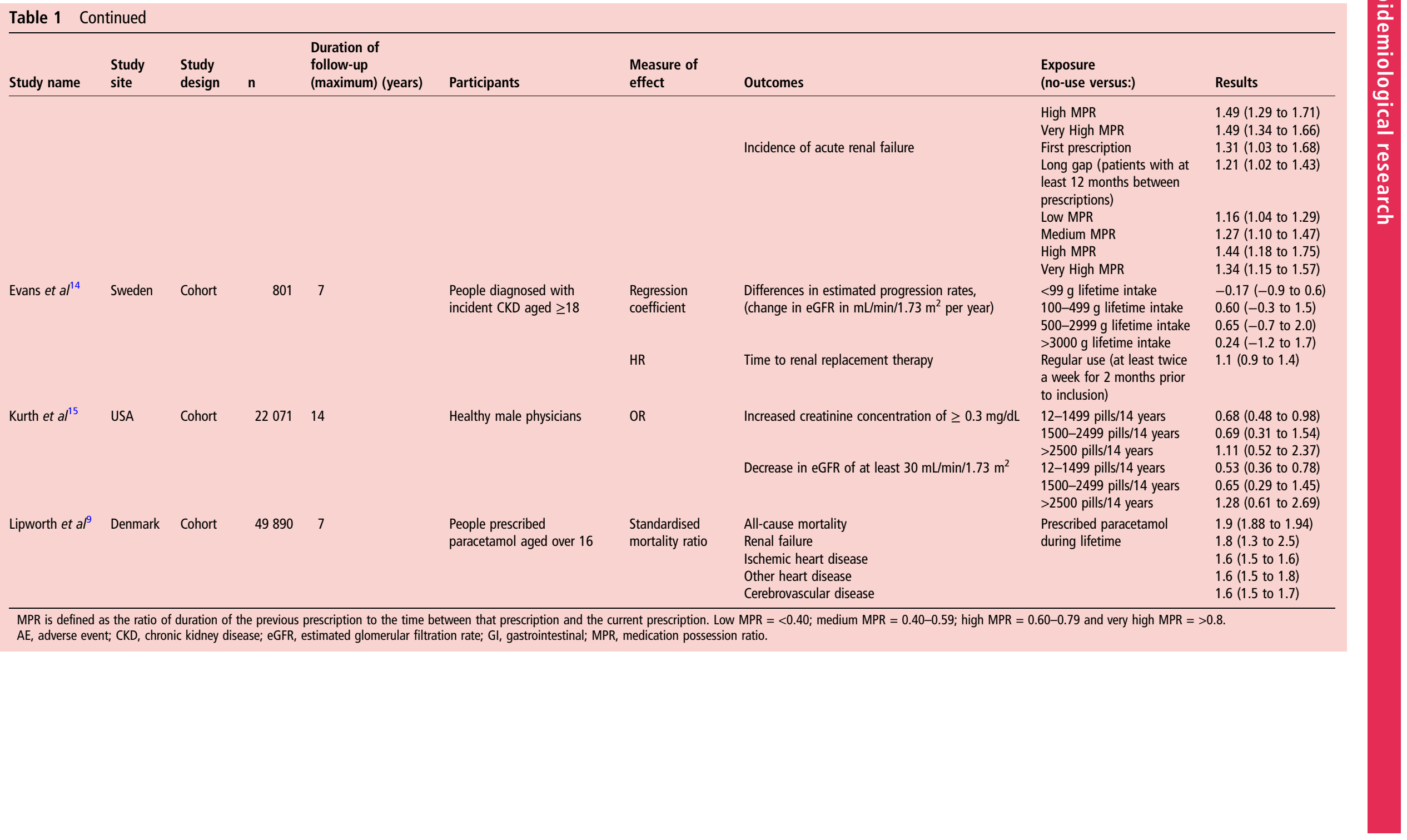




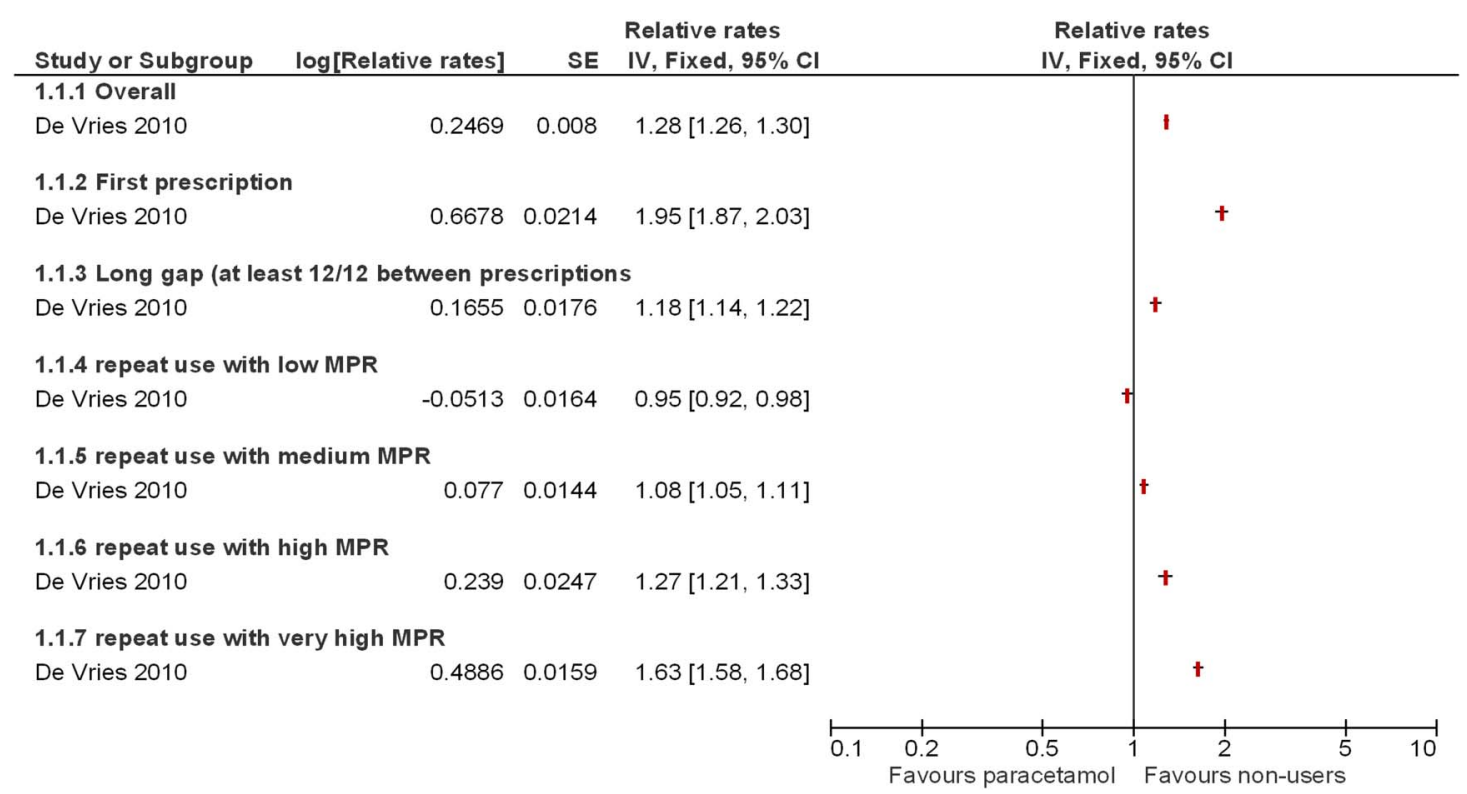

Figure 2 Mortality. The relative rate of all-cause mortality in patients taking paracetamol versus patients not taking paracetamol. The online supplementary material provides the references for the included studies.

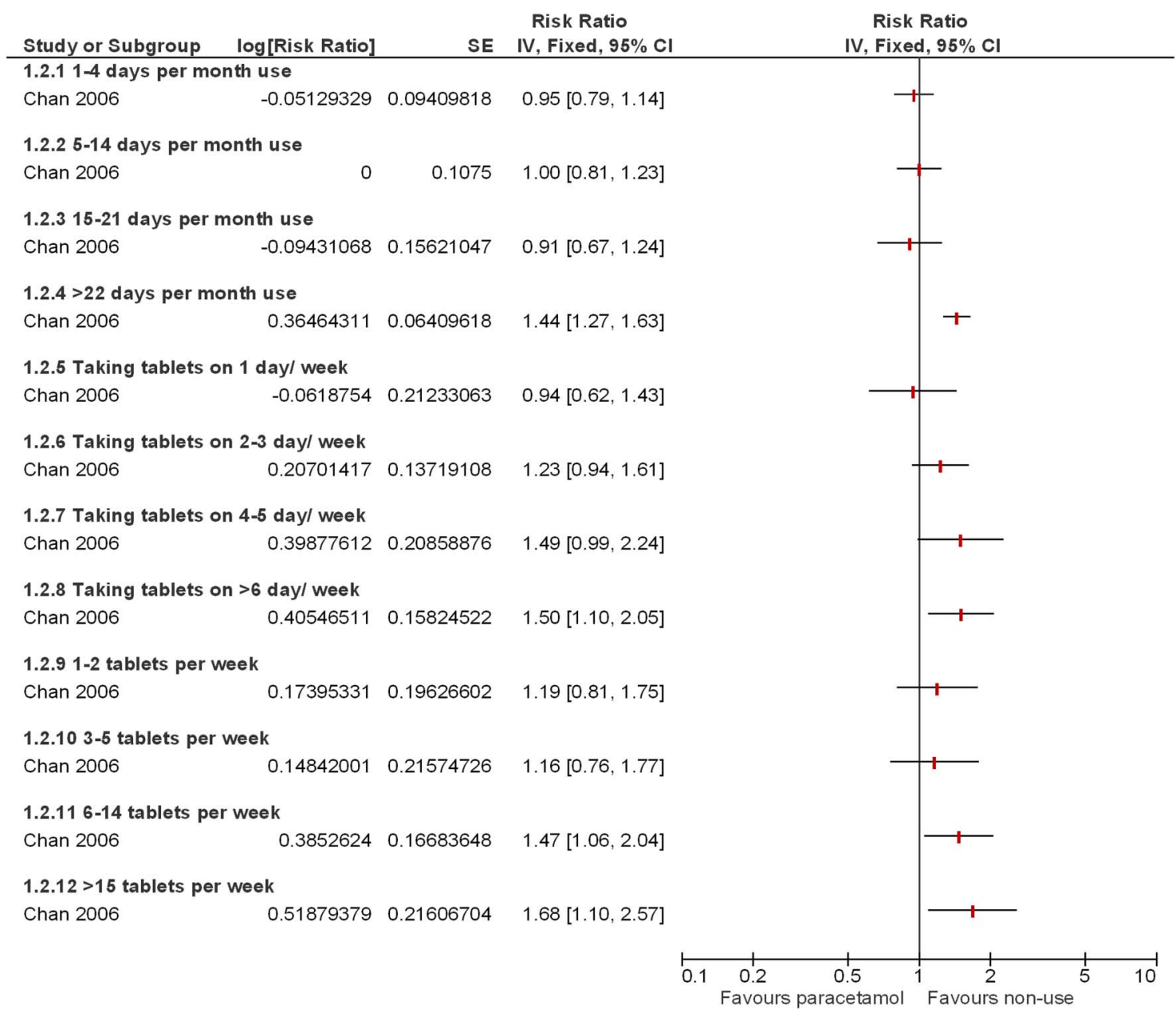

Figure 3 Cardiovascular adverse events (AEs). The risk ratio of cardiovascular AEs (confirmed or probable non-fatal myocardial infarction, non-fatal stroke, fatal coronary heart disease or fatal stroke) in patients taking paracetamol versus patients not taking paracetamol. The online supplementary material provides the references for the included studies. 


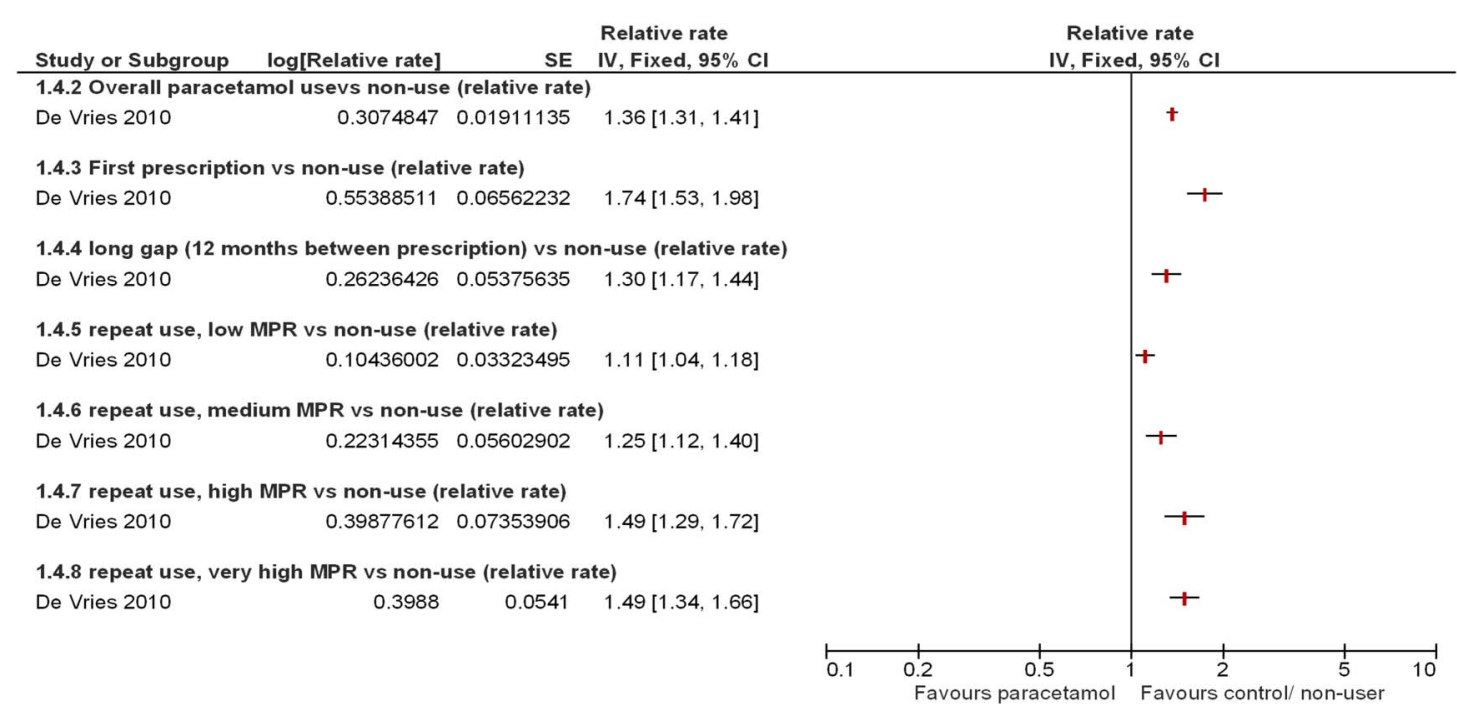

Figure 4 Gastrointestinal adverse events (GI AEs). The relative rate of upper GI AEs (gastroduodenal ulcers and complications such as upper GI haemorrhages) in patients taking paracetamol versus patients not taking paracetamol. The online supplementary material provides the references for the included studies.

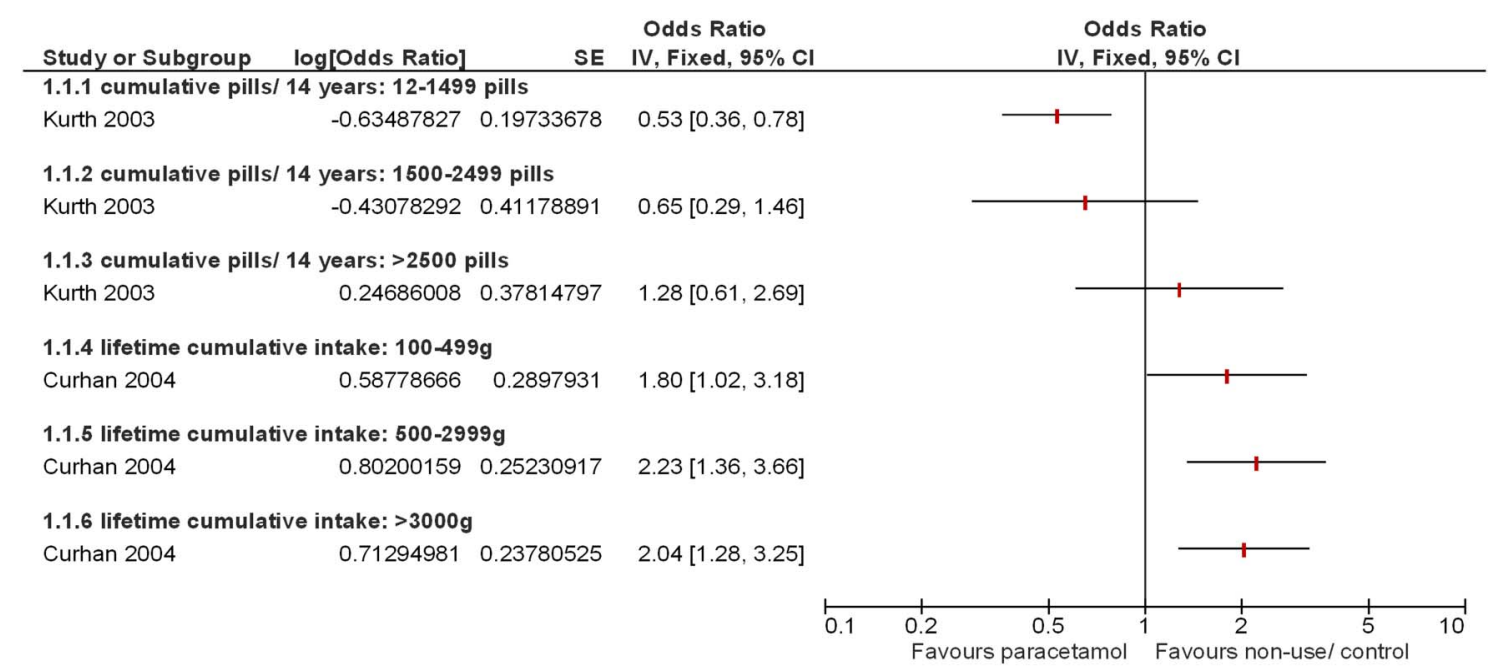

Figure 5 OR of a decrease in estimated glomerular filtration rate of at least $30 \mathrm{~mL} / \mathrm{min} / 1.73 \mathrm{~m}^{2}$ in patients taking paracetamol versus patients not taking paracetamol. The online supplementary material provides the references for the included studies.

been recently suggested for paracetamol in the treatment of OA joint pain ${ }^{4}$ and low back pain, more careful consideration of its usage is required. ${ }^{20}$ Prescribers need to be aware of patients' individual responses to paracetamol and the observed increased toxicity with regular and higher dosing within standard analgesic dose ranges. Based upon the data presented above, we believe the true risk of paracetamol prescription to be higher than that currently perceived in the clinical community. Given its high usage and availability as an over-the-counter analgesic, a systematic review of paracetamol's efficacy and tolerability in individual conditions is warranted.

\footnotetext{
Author affiliations

${ }^{1}$ South London and the Maudsley Mental Health Trust, Maudsley Hospital, London, UK ${ }^{2}$ National Clinical Guideline Centre, London, UK

${ }^{3}$ Division of Rheumatology, Orthopaedics and Dermatology, Clinical Sciences Building, City Hospital, Nottingham, UK

${ }^{4}$ Northumbria Healthcare NHS Foundation Trust, Newcastle University, Ashington, UK ${ }^{5}$ Research Institute for Primary Care and Health Sciences, Keele University, Keele, UK

${ }^{6}$ Arthritis Research UK Primary Care Centre, Research Institute for Primary Care \& Health Sciences, Keele University, Keele, UK
}

${ }^{7}$ Ealing Hospital NHS Trust Community Musculoskeletal Service, Clayponds Hospital, London, UK

${ }^{8}$ Gordon House Surgery, London, UK

${ }^{9}$ Encompass Healthcare, Washington, Tyne and Wear, UK

${ }^{10}$ Leeds Institute of Rheumatic and Musculoskeletal Medicine, University of Leeds and NIHR Leeds Musculoskeletal Biomedical Research Unit, Leeds UK

Acknowledgements The authors would like to acknowledge the following individuals for their help in the preparation of this work: Ms Erika Baker, Cheshire Merseyside Commissioning Support Unit. Ms Jo Cumming, Arthritis Care UK. Mr Richard Frearson, Newcastle Upon Tyne Hospitals NHS Foundation Trust. Mr Peter Kay, Wrightington Hospital. Mr Brian Lucas, Kings Lynn NHS Foundation Trust. Mr Robert Middleton, Royal Bournemouth Hospital. Mr Anthony Whiting. No compensation was received for any contribution.

Contributors All authors fulfil the International Committee of Medical Journal Editors (ICMJE) criteria for authorship credit. ER contributed to the study design, collected, interpreted, analysed the data, drafted and edited the manuscript. VDN contributed to the study design, collected, interpreted and analysed the data and edited the manuscript. SB screened the literature search results (titles and abstracts) for relevant articles; screened relevant full-text articles for inclusion criteria; extracted study characteristics and summary statistics with $95 \%$ Cls for all studies included in the review; recorded the data in standard form; and assessed the study quality using GRADE. SL provided management support to research fellow staff and contributed to 
the writing of this article. MC contributed to the study design, analysed data and edited the manuscript. PM conducted the search for the systematic review and complied the references for the manuscript. MD contributed to study design, data interpretation and editing of final paper. WZ contributed to study design, data interpretation and editing. FB contributed to study design and edited the manuscript. MP contributed to (i) defining the question for the systematic review, (ii) analysing and interpreting data and (iii) writing the paper (commented on all drafts of the paper). KD was a member of the group that conceived the manuscript and was involved in reviewing versions of the manuscript for publication. IB contributed to data interpretation and writing. EW contributed to study design and editing of the manuscript. PC contributed to study initiation, design, analysis, interpretation and writing, as well as reviewing the final version of the manuscript.

Funding The review was undertaken by the National Clinical Guidelines Centre, UK.

Competing interests None.

Provenance and peer review Not commissioned; externally peer reviewed.

Data sharing statement PC and ER had full access to all the data in the study and take responsibility for the integrity of the data and the accuracy of the data analysis. There is no additional unpublished data from this study.

Open Access This is an Open Access article distributed in accordance with the Creative Commons Attribution Non Commercial (CC BY-NC 4.0) license, which permits others to distribute, remix, adapt, build upon this work non-commercially, and license their derivative works on different terms, provided the original work is properly cited and the use is non-commercial. See: http://creativecommons.org/ licenses/by-nc/4.0/

\section{REFERENCES}

1 Blieden M, Paramore LC, Shah D, et al. A perspective on the epidemiology of acetaminophen exposure and toxicity in the United States. Expert Rev Clin Pharmacol 2014:3:341-8.

2 Jordan KM, Arden NK, Doherty M, et al. EULAR Recommendations 2003: an evidence based approach to the management of knee osteoarthritis: Report of a Task Force of the Standing Committee for International Clinical Studies Including Therapeutic Trials (ESCISIT). Ann Rheum Dis. 2003;62:1145-55.

3 Hinz B, Brune K. Paracetamol and cyclooxygenase inhibition: is there a cause for concern? Ann Rheum Dis 2012;71:20-5.

4 Zhang W, Nuki G, Moskowitz RW, et al. OARSI recommendations for the management of hip and knee osteoarthritis: part III: Changes in evidence following systematic cumulative update of research published through January 2009 Osteoarthritis Cartilage 2010;18:476-99.
5 Stroup DF, Berlin JA, Morton SC, et al. Meta-analysis of observational studies in epidemiology. JAMA 2000;283:2008-12.

6 Modher D, Liberati A, Tetzlaff J, et al.; PRISMA group. Preferred reporting items for systematic reviews and meta-analyses: the PRISMA statement. J Clin Epidemiol 2009;62:1006-12.

7 von Elm E, Altman DG, Egger M, et al.; TROBE Initiative. Strengthening the Reporting of Observational Studies in Epidemiology (STROBE) statement: guidelines for reporting observational studies. BMJ 2007;335:806-8.

8 de Vries F, Setakis E, van Staa TP, et al. Concomitant use of ibuprofen and paracetamol and the risk of major clinical safety outcomes. $\mathrm{Br} J$ Clin Pharmacol 2010;70:429-38.

9 Lipworth L, Friis S, Mellemkjaer L, et al. A population-based cohort study of mortality among adults prescribed paracetamol in Denmark. J Clin Epidemiol 2003; 56:796-801.

10 Chan AT, Manson JE, Albert CM, et al. Nonsteroidal anti-inflammatory drugs, acetaminophen, and the risk of cardiovascular events. Circulation 2006;113:1578-87.

11 Curhan GC, Willett WC, Rosner B, et al. Frequency of analgesic use and risk of hypertension in younger women. Arch Intern Med 2002;162:2204-8.

12 Dedier J, Stampfer M, Hankinson S, et al. Nonnarcotic analgesic use and the risk of hypertension in US women. Hypertension 2002;40:604-8.

13 Curhan GC, Knight EL, Rosner B, et al. Lifetime nonnarcotic analgesic use and decline in renal function in women. Arch Intern Med 2004;164:1519-24.

14 Evans M, Fored CM, Bellocco R, et al. Acetaminophen, aspirin and progression of advanced chronic kidney disease. Nephrol Dial Transplant 2009;24:1908-18.

15 Kurth T, Glynn RJ, Walker AM, et al. Analgesic use and change in kidney function in apparently healthy men. Am J Kidney Dis 2003;42:234-44.

16 Garcia Rodriguez LA, Hernandez-Diaz S. Relative risk of upper gastrointestinal complications among users of acetaminophen and nonsteroidal anti-inflammatory drugs. Epidemiology 2001;12:570-6.

17 Rahme E, Pettitt D, LeLorier J. Determinants and sequelae associated with utilization of acetaminophen versus traditional nonsteroidal antiinflammatory drugs in an elderly population. Arthritis Rheum 2002;46:3046-54.

18 Rahme E, Barkun A, Nedjar H, et al. Hospitalizations for upper and lower Gl events associated with traditional NSAIDs and acetaminophen among the elderly in Quebec, Canada. Am J Gastroenterol 2008;103:872-82.

19 Doherty M, Hawkey C, Goulder M, et al. A randomised controlled trial of ibuprofen, paracetamol or a combination tablet of ibuprofen/paracetamol in community-derived people with knee pain. Ann Rheum Dis 2011;70:1534-41.

20 Williams CM, Maher CG, Latimer J, et al. Efficacy of paracetamol for acute low-back pain: a double-blind, randomised controlled trial. Lancet 2014;384:1586-96. 